\title{
Measuring Labor Supply and Demand Shocks during COVID-19
}

\author{
Tania Chaar $^{1} \cdot$ Charles Bromwich $^{1}$ (D)
}

Accepted: 17 December 2021 / Published online: 7 January 2022

(C) International Atlantic Economic Society 2022

JEL J40 $\cdot$ J20

The COVID-19 pandemic has resulted in significant disruption to the Canadian labour market. In the initial stage of the pandemic, Canadian authorities enforced containment measures to curb the pandemic's impact within Canada. Following new waves of cases, public health authorities shifted their approach to instill more targeted and less general restrictive measures. The supply and demand effects caused by these measures had a heterogeneous impact on different sectors of the labour market. This study measures how labour supply and demand shocks affected different sectors of the Canadian economy, how the shocks varied among the sectors and how those shocks changed as the pandemic progressed. These results offer important insights to decision-makers in both fiscal and monetary policy, which could help them respond in a more targeted way to future pandemics. Since lockdown measures had heterogeneous effects on different sectors, a policy designed to support demand may have affected a supply-constrained sector differently than a sector not facing supply constraints.

A COVID-19 demand and supply shock analysis was performed by estimating labour demand and supply shocks at the North American Industry Classification System (NAICS) two-digit level. The complete list of sectors studied is available in Online Supplemental Appendix Table 1. This study's approach was inspired by Brinca et al. (Federal Reserve Bank of St Louis Working Paper, 2020, https://

\footnotetext{
Supplementary Information The online version contains supplementary material available at
} https://doi.org/10.1007/s11293-021-09730-5.

Charles Bromwich

charles.bromwich@umontreal.ca

1 University of Ottawa, Ottawa, ON K1N 6N5, Canada 
doi.org/10.20955/wp.2020.011). Their paper applied the methodology proposed by Baumeister and Hamilton (Econometrica, 2015), in which a Bayesian structural vector autoregressive model was used to analyze the shock dynamics of monthly wages and hours worked for each sector of the U.S. economy. The current study sought to replicate this research in a Canadian context. This methodology uses sign restrictions on labour supply and demand elasticities, assuming that the labour demand curve should be downward sloping and that the labour supply curve should be upward sloping. The study also made explicit assumptions about the probability distribution of the slopes of the labour supply and demand curves for each sector which were informed by Lichter et al. (IZA Discussion Paper No. 7958, 2014, https://docs.iza.org/dp7958.pdf) as well as Jantti et al. (Journal of Public Economics, 2020). This study's results are framed by identifying five main periods in the progression of the COVID-19 pandemic. These stages included the first wave (March 2020 to April 2020), the reopening (May 2020 to August 2020), the second wave (September 2020 to January 2021), the end of the second wave (February 2021 to March 2021) and the third wave (April 2021 to May 2021).

Analysis of the first wave of the pandemic strongly suggests that the initial decrease in employment in Canada was primarily the result of a strong negative supply shock affecting certain sectors, notably food and accommodation services, information, and construction, as demonstrated in Online Supplemental Appendix Figs. 1 and 2. Another notable result from this period was the absence of an effect in other sectors, such as public administration and professional, scientific and technical services. This heterogeneity was present throughout the entirety of the period under study, with some sectors having an outsized effect on changes to aggregate employment numbers.

The second period of the pandemic analysed herein, the reopening in the summer of 2020, saw significant recoveries occur in the sectors that were affected by the first wave of the pandemic (Online Supplemental Appendix Figs. 3 and 4). Reduced restrictions allowed non-essential businesses to open their doors, leading to significant increases in employment. Supply shocks also dominated in this period, with the construction, food and accommodation services, and information sectors experiencing the largest positive shocks.

The second wave of the pandemic offered an opportunity to see how the shocks differed from the first wave (Online Supplemental Appendix Figs. 5 and 6). First, the dominance of supply shocks present during the first stages of the pandemic lessened during the second wave of the pandemic, with many sectors affected by supply and demand shocks of comparable size. Furthermore, some sectors that were previously severely affected by pandemic restrictions saw positive supply shocks during the second wave, such as construction. This could be seen as evidence that some sectors became more resilient to restrictions through improved health and safety measures, or workers became more willing to return to work in the second wave. Subsequent periods that were analysed showed similar trends, with mixed shocks that seem less tied to COVID-19 restrictions, and more closely tied to the state of the economy.

The heterogeneous nature and magnitude of COVID-19 effects posed challenges to this empirical analysis. Large shocks can make the residuals non-stationary. The 
assumption of linearity can be questioned as the magnitude of the shock can cause large shifts in supply and demand curves when the elasticities are no longer constant. Finally, fluctuations in sectoral wages could stem from a change in the composition of occupations, exaggerating the importance of supply shocks. When correcting for this composition effect, Brinca et al. (2020) found no significant change in the results.

Overall, these results suggest that the pandemic's effect on economic sectors within Canada was highly heterogeneous during the first wave of the pandemic, and only became more so during subsequent waves of the virus. Supply shocks were found to be the principal factor in changes to hours worked during the first wave of the pandemic, though this effect became weaker over time. Finally, subsequent waves of the pandemic had less of an impact on the changes to hours worked.

Publisher's Note Springer Nature remains neutral with regard to jurisdictional claims in published maps and institutional affiliations. 